PILAR

AUMENTE-RIVAS
Profesora Emérita de la Universidad Complutense de Madrid aumenterivas@gmail.com

\title{
Arte colaborativo y Distritos culturales. Revisitando algunas fuentes
}




\section{ABSTRACT}

The reflection on the collaborative calls for a review of the contemporary roots of the concept (Kropotkin) and its development (Ostrom). It is the core of our study. Another section is dedicated to some theorists of Cultural Districts (Sacco, Ferilli) who seem to make possible the passage from the "isolated one-subject" to the "we-working together-collective", reviewing their level of differentiation. The objectives of the study are: to characterize the scope of the collaborative through its sources; select some of the main notions of the collaborative universe; revisit them highlighting features provided; point out aspects of the current theory of the Districts, in order to the compatibility or discrepancy with the collaborative. The theoretical focus of the research calls for a methodology based on the management of the legacy of knowledge that supports collaborative actions, as well as the development of cultural districts. Sequencing supposes rereading in historical development of source texts of key, founding and current collaborative theories; selection of fragments that characterize the identifying notions; choice of theorists of Cultural Districts that allow a suitable analysis with respect to objectives; critical presentation of the theoretical aspects of compatibility or disparity from the theory of Districts, in relation to the collaborative world.

\section{Keywords}

Cultural districts; theoretical sources of the collaborative field; collaborative cultural practices; collaborative art

\section{RESUMEN}

La reflexión sobre lo colaborativo insta a la revisión de las raíces contemporáneas del concepto (Kropotkin) y su desarrollo (Ostrom). Es el núcleo de nuestro estudio. Otra sección está dedicada a algunos teóricos de Distritos Culturales (Sacco, Ferilli) que parecen posibilitar el paso del "uno-sujeto aislado", al "nosotros-trabajando juntos-colectivo", Revisando su nivel de diferenciación. Los objetivos del estudio son: caracterizar el ámbito de lo colaborativo a través de sus fuentes; seleccionar algunas de las principales nociones del universo colaborativo; revisitarlas destacando características aportadas; señalar aspectos de la teoría actual de los Distritos, en orden a la compatibilidad o discrepancia con lo colaborativo. El enfoque teórico de la investigación insta a una metodología basada en el manejo del legado de conocimientos que sustenta las acciones colaborativas, así como el desarrollo de los distritos culturales. La secuenciación supone relectura en desarrollo histórico de textos-fuente de teorías colaborativas clave, fundadoras y actuales; selección de fragmentos que caractericen las nociones identificativas; elección de teóricos de Distritos Culturales que posibiliten un análisis idóneo respecto a objetivos; presentación crítica, de los aspectos teóricos de compatibilidad o disparidad desde la teoría de los Distritos, en relación al mundo colaborativo.

\section{Palabras clave}

distritos culturales; fuentes teóricas del ámbito colaborativo; prácticas culturales colaborativas; arte colaborativo 


\section{INTRODUCCIÓN}

Los Distritos Culturales nacieron para paliar la crisis económica surgida tras el vaciamiento industrial de los países occidentales. Esta migración industrial dejó muy afectadas a las ciudades que habían sido sede de las grandes industrias, auténticos motores de la economía en la primera mitad del siglo XX. Fueron impulsados, en un primer momento, por instituciones oficiales vinculadas a la imprescindible revitalización urbana. Su desarrollo ha venido apoyado en la expansión de las industrias culturales, lo que ha conllevado la crítica desde un primer momento (Horkheimer y Adorno) y que se ha mantenido y acrecentado (Garnham, Bustamante, entre otros) desde entonces. Hoy algunos de los distritos culturales surgen por iniciativa de bases más próximas a la ciudadanía, corresponde por tanto hacer una revisión que afronte la valoración de su pertinencia en un mundo en que las prácticas del hacer en colaboración han transformado las posibilidades del vivir en el mundo.

Por otro lado, la reflexión sobre lo colaborativo insta a la revisión de las raíces contemporáneas del concepto, así como a la frecuentación de fuentes provenientes de distintos ámbitos, que han ido tejiendo el lecho que sustenta la posibilidad de penetración de las prácticas artísticas colaborativas en el ámbito de lo urbano y especialmente en el entorno de los Distritos Culturales.

La década de los años 60 fue muy floreciente en ensayos de prácticas artísticas con el acento puesto en la valoración de la presencia del espectador, así como en la potenciación de los grupos como factor de producción artística. Ya a finales de los años 70, se produce un cambio en la primacía del pensamiento. El individualismo ejercerá desde ese periodo una gran presión y buscará barrer los avances de los movimientos que las colectividades habían logrado realizar en décadas anteriores. Desde los noventa, sin embargo, se reinstaurará con fuerza la búsqueda de un mundo en el que, frente al "uno-individuo", se instale el "nosotros-colectivo". De la elaboración de objetos (obras de arte), "los colectivos pasaron a gestar relaciones sociales a través de intervenciones mediáticas, instalaciones y performances” (López, 2016, p.17).

La oposición de estos términos se formalizó ya en el siglo XIX en pleno desarrollo industrial. Teorías como las de Marx y Engels, pero también las de Kropotkin, ponen sólidas bases teóricas que Althusser, Lefebvre, Harvey, Ardenne, y bajo la orientación de Elinor Ostrom, muchos más, irán desarrollando, desde la segunda mitad del siglo XX un caldo de cultivo favorable a ese cambio hacia la consolidación de las prácticas colectivas. Lo irán haciendo a través de sus respectivas posiciones teóricas, que no siempre coincidirán con una línea marxista ortodoxa declarada, ni anarquista reconocida, sino, en ocasiones, de una izquierda sin etiquetas. A veces, se referirán únicamente de forma parcial al núcleo de la reflexión, como en el caso de Merleau-Ponty o Habermas, para desbrozar el camino hacia consolidación de la arribada de lo colectivo y, finalmente, del trabajo colaborativo que, en parte a través del éxito de las redes (Benkler), ha ido introduciendo un cambio en la sociedad que quiere verse plasmado asimismo en el ámbito de lo urbano. 


\section{DESARROLLO}

\subsection{Bases teóricas del ámbito colaborativo}

\subsubsection{Nociones fundamentadoras}

El concepto de unidad toma fuerza indubitable a través del mandato marxiano. El papel jugado por las apelaciones de Marx y Engels a la unión de los obreros para luchar por la consecución de una mejor situación de clase es indiscutiblemente una base fundadora para la fortuna del concepto que se resuelve en la acción de una colectividad. Las llamadas dispersas de Marx en EI Capital se materializan en el cierre del Manifiesto Comunista de Marx y Engels con la invocación: “iiiProletarios del mundo, uníos!!!" (Marx y Engels, 2015, p.38).

Ahora bien, no solo exaltaban la unión de los elementos individuales sino la unión entre agrupaciones e incluso entre partidos (ibidem). Althusser en su obra dedicada a la interpretación de Marx (Althusser y Balibar, 2019, p. 262 y ss,) subraya la dedicación de Marx a lo colectivo, al trabajador colectivo que Marx denomina productor de trabajo social.

¿Por qué medio se ha de conseguir el éxito de la tarea del comunismo? En el mismo Manifiesto se identifica el medio: la violencia "sus objetivos sólo pueden alcanzarse derrocando por la violencia todo el orden social existente. Tiemblen, si quieren, las clases gobernantes, ante la perspectiva de una revolución comunista" (Marx y Engels, 2015, p.38-39). Porque "el proletariado...no puede levantarse, incorporarse, sin hacer saltar, hecho añicos desde los cimientos hasta el remate, todo ese edificio que forma la sociedad oficial (Marx y Engels, 2015, p.21). Al fin y al cabo: "Toda la historia de la sociedad humana, hasta la actualidad, es una historia de luchas de clases... (Marx y Engels, 2015, p.12). Los conceptos de violencia, enfrentamiento y lucha aparecen como vía imprescindible para el logro de un mundo mejor "se enfrentan la masa del pueblo, los obreros, como no propietarios, y los no obreros como propietarios de estos medios de producción" (Marx, 1870, Posic 611).

Recordemos, no obstante, que estas luchas tienen como primer objetivo el reforzamiento de la "unión obrera" para la preparación en "previsión de posibles batallas". (Marx y Engels, 2015, p. 19). Aquel mandato de unión lanzado en el Manifiesto tuvo sus repercusiones inmediatas, como la creación en 1864 de la "Asociación Obrera Internacional" (Asociación internacional de trabajadores, AIT, llamada: Primera Internacional) en la que participaron Marx, Engels y también Bakunin. Hay que tener en cuenta asimismo que Bakunin había organizado unos años antes la "Asociación Internacional Revolucionaria" (Dolgoff, 2017), conocida como "Fraternidad Internacional" y más tarde, en 1868 fundó la "Alianza Internacional de la Democracia Socialista" con el objetivo de competir con Marx, lo que derivó, tras un periodo de tensiones, en la expulsión de Bakunin de la Asociación obrera en el año 1872. Quedaban separados los caminos de comunistas (marxistas de la I Internacional) y anarquistas (Bakuninistas) que se agruparán entorno a la Internacional de Saint Imier (1872).

Bakunin es la figura más representativa del anarquismo colectivista, caracterizado, asimismo, por el socialismo libertario, y el confederalismo regionalista. Buscaba la destrucción del poder jerarquizado (el Estado y sus estructuras) y el desarrollo de una sociedad basada en la acción de colectivos, dueños de los medios de producción, organizados en federaciones. "La organización federal, las asociaciones obreras, de grupos, de comunas, de cantones y en fin de regiones y de pueblos, es la única condición para una libertad verdadera y no ficticia" (Bakunin, 1873, 
posic.14), esa "libertad democrática que debe ser fundamento de la paz" (Bakunin, 1868, p.104). Es decir, fundar la paz sobre la democracia y la libertad. Condición sine qua non puede conseguirse aquella. Fue defensor de la creación de los "estados unidos de Europa" (Bakunin, 1868, posic.173) y proponía la abolición de las fronteras y el derecho histórico de los Estados (ídem: 188)

Señaló la importancia de la educación y las diferencias entre las dos principales categorías, la de la clase burguesa y la de "las clases obreras desheredadas tanto del Capital como de la tierra, y privadas de toda educación y de toda instrucción". (Bakunin,1868, p. 252) que se resolvería con la "reforma radical en las condiciones económicas actuales de las clases obreras" (Bakunin, 1868, p.269). Puso en primer plano la importancia del tiempo de ocio reivindicando su implantación en el ámbito de trabajo de la clase obrera: "ratos de ocio, esa condición suprema de todo humano desenvolvimiento, tanto intelectual como moral, condición que no se realiza jamás para las clases obreras" (Bakunin, 1868, p.315).

Entiende el Estado solo como regulador de la sociedad, el cual, renunciando a su papel represor, se convertiría según Proud'hom en un "servicio subalterno" a la sociedad. (Bakunin, 1868, p. 1644). Su rechazo se extendía a todo tipo de autoridad: "No reconozco ninguna autoridad infalible" (Dolgoff, 2017 p. 3525), "rechazamos toda legislación, toda autoridad y todos los poderes privilegiados, sancionados, oficiales y legales. "En consecuencia, no tengamos legislación externa ni autoridad (Dolgoff, 2017, p.3518).

Desde filas revolucionarias de orientación anarquista, Kropotkin elaboró su teoría del "Apoyo Mutuo" como factor de la evolución humana, en contraposiciòn a las teorías de la lucha: "podemos afirmar que el principal papel en la evolución ética de la humanidad fue desempeñado por la ayuda mutua y no por la lucha mutua". (Kropotkin, 2016, p.352). Señala esta noción como característica siempre presente, aunque no reconocida en todo momento, como fuente de progreso:

\section{La necesidad de ayuda y apoyo mutuos que se ha refugiado quizá en el círculo estrecho de la familia, entre los vecinos de las calles y callejuelas más pobres, en la aldea o en las uniones secretas de los obreros, renace de nuevo incluso en nuestra sociedad moderna..." y "siempre ha sido, el principal impulsor en el camino del progreso futuro. (Kropotkin, 2016, p.344)}

Sin embargo, mientras Marx y Engels piden la unión para hacer efectiva la lucha de clases, en el caso de Kropotkin los sentimientos de solidaridad humana están fuertemente arraigados en la conciencia y el corazón de los hombres (Ibidem), lo que se resuelve en la idea de unidad con todo ser humano y en la idea de igualdad de derecho general (Idem, p.352). La solidaridad y el concepto de ayuda mutua es reconocida también entre las clases pudientes, en el ámbito de sus propios niveles y no la totalidad de capas sociales entre sí, por lo que se entendían como elementos de clase. Ahora bien, su distinción principal se posa en que, en la evolución ético-social de la humanidad, la solidaridad, la ayuda mutua es el principal factor, frente a la propuesta que defiende la lucha mutua. No se trata tanto de una posición antimarxiana, aunque resulte serlo, es un posicionamiento a favor de Darwin y contra la mala interpretación que, según Kropotkin, Thomas $\mathrm{H}$. Huxley había hecho de las teorías darwinianas en la obra La lucha por la existencia en la sociedad humana (1888).

El papel de Malthus como antecedente teórico de esa lucha por la existencia debe ser, al menos, 
mencionado (https://museo-etnografico.com/pdf/puntodefuga/171128malthus), al igual que el desempeñado por Karl F. Kessler en la orientación del apoyo mutuo, quien en 1879 leyó en público el texto "Sobre la ley de la ayuda mutua" en el que defendía la teoría de la ayuda mutua frente a la teoría Darwiniana de la competición. Acusó a Darwin de dejar de lado el concepto de cooperación y señaló la importancia de los dos niveles sin exclusión para el desarrollo de la vida: "No rechazo la lucha por la existencia, sólo afirmo que el desarrollo progresivo, tanto de todo el reino animal y, sobre todo, de la humanidad no es facilitado por la lucha mutua tanto como por la ayuda mutua". (Molinero, 2015).

Estas afirmaciones impulsaron las reflexiones de Kropotkin que, sin atacar la noción de selección natural, las orientará en otra dirección sociopolítica, reivindicando la interpretación de "los más aptos "que no tienen porqué ser los más fuertes" sino los que mejor se adaptan al medio. La sociedad humana evoluciona y se afianza a través del apoyo y la ayuda mutua y no a través de la lucha (Kropotkin, 2016, p. 352).

\subsubsection{Fuentes y apoyos teóricos para el reconocimiento del otro}

Casi al tiempo, en los albores del S. XX, la fenomenología de Husserl pone en marcha un recurso gnoseológico: la reducción o epojé (Husserl, 1982, p.55) y también propone el concepto de intersubjetividad en la quinta de las Meditaciones Cartesianas, donde afirma que cada sujeto trascendental no solo está "al lado de los otros" (beieinander), y "con los otros" (miteinander) sino "en los otros" (ineinander). De manera simple, podemos decir que es el proceso recíproco por el que se comparte la conciencia y conocimiento de una persona a otra. Lo cual supone el reconocimiento del otro y la posibilidad el proceso de conocimiento en red, en otras vertientes se interpretará como la disponibilidad a la comprensión de acuerdos, así como la de comprensión de divergencias (perspectivas psicológicas).

La obra de arte será objeto de examen por parte de Husserl, buscando el conocimiento del verdadero sentido de una obra de arte en general y el sentido particular de una obra de arte determinada. En el primer caso estudiamos la esencia de una obra de arte en pura universalidad; en el segundo caso, el contenido de la obra de arte efectivamente dada, según sus determinaciones verdaderas (Husserl, 1982, p.110). Husserl subraya el carácter relacional de la fenomenología que procede aclarando visualmente, determinando y distinguiendo el sentido. “Compara, distingue, enlaza, pone en relación" (Husserl, 1982, p. 71).

Los discípulos de Husserl, algunos de ellos heterodoxos y hasta" heréticos" con su teoría, afianzarán e incluso reforzarán la tendencia relacional de sus presupuestos. En la nómina de la escuela fenomenológica francesa, destacan, estricto senso, fundamentalmente, Merleau-Ponty y Dufrenne. Merleau, que dedicó un gran estudio a la percepción, haciendo bascular el interés teórico hacia el perceptor-espectador, en el ámbito de estudio del cuerpo como expresión, abre la puerta al reconocimiento del otro "Es por mi cuerpo que comprendo al otro, como es por mi cuerpo que percibo "cosas" (Merleau-Ponty, 1975, p. 203). Es decir, que aborda con empeño la relación con el otro, que se presenta como necesario, incluso para que el uno se realice.

Según Merleau-Ponty, Marx capta lo que es, en esencia, el ser humano (una subjetividad intersubjetiva; nunca un ser aislado o absoluto, sino ya comprometido en origen) y lleva esta intuición a un intento de transformación del mundo en consonancia con ella. (Peñaranda, 1992, p. 235). Siguiendo a Marx, el hombre, según Merleau-Ponty, «existe» y esta existencia... es 
ante todo coexistencia (Revault d'Allones, 2001, p. 573). Merleau-Ponty insiste en (Sens et non Sens): el sistema de la coexistencia humana compromete al hombre en el mundo, respecto a los objetos, las cosas y al otro (Revault d'Allonnes, 2001, p. 573).

En su teoría es el quiasmo el que nos permite convertirnos en los demás en una relación primitiva. Salimos de nosotros mismos y llegamos a ser el mundo y el otro. De sumo interés para el objetivo de la investigación resultan unas palabras escritas en Fenomenología de la Percepción: "nous sommes l'un pour l'autre collaborateurs dans une reciprocité parfaite, nos perspectives glissent l'une dans l'autre, nous coexistons á travers un mème monde. (fragmentos de Fenomenología de la Percepción. En 1971, p.186).

Por otro lado, Husserl, el gran maestro de Merleau-Ponty, había señalado la existencia de una crisis de la idea de verdad. Merleau-Ponty, deduce en consecuencia, que si de nada podemos estar seguros, no lo podemos estar tampoco de la lucha de clases, y ante los acontecimientos que se sucedieron en la práctica marxista, valora la situación como inaceptable práctica comunista "demasiado grave como para seguir apoyando al comunismo actual por más tiempo (en diversa obras entre las cuales "(Pintos,1992, p. 240), por ello aboga por el "A-comunismo y por una revisión y crítica de los postulados marxianos que lo permitieron (Pintos, 1992, p. 241). Es decir, Merleau deriva hacia una situación de puesta en entredicho de la conciencia de clase. Esa conciencia debe ser formada y corregida (Pintos, 1992, p. 247).

Igualmente cuestiona la violencia a través del cuestionamiento de la revolución "Las revoluciones son verdaderas como movimientos y falsas como regímenes» (Pintos, 1992, p. 249). Al final de Las Aventuras de la Dialéctica. (1974, p. 228 y ss. especialmente 256-257), hace ver la necesidad de dejar el marxismo y situarse en la "tercera vía" (Revault d'Allones, 2001. posc. 1356, 1360 y 1363) de una izquierda situada entre el proletariado y el orden del capitalismo. Es decir, Merleau-Ponty se instala en un pensamiento de izquierdas opuesto a la lucha de clases y al enfrentamiento violento.

Mikel Dufrenne enlaza con la línea de Merleau y desarrolla en el ámbito estético el estudio del papel del tercer elemento en la triada constituyente de la experiencia estética: artista -obra de arte-espectador y trabajó en el reconocimiento del papel del espectador como co-creador. El espectador no es solamente el testigo que consagra la obra, es, a su manera, el ejecutante que la cumple; el objeto estético (obra de arte) necesita de él para aparecer (Dufrenne, 1981, p.112), (Aumente, 1985, p. 235). No se trata de una actividad productiva, convertir la obra en objeto estético "no es crearla, aunque sea cumplirla, elevarla a su verdadero ser", pero... "puede ser recrearla" (Dufrenne,1981, p. 133 y 134).

En el mundo contemporáneo el artista da a conocer sus técnicas libremente frente al ocultismo histórico, y en ello ve el teórico una invitación a la participación y en ciertos casos lo considera una llamada a la participación colectiva (Dufrenne,1981, p.134). Recordando a Duvignaud (Fêtes et Civilitations) menciona como el arte de nuestra civilización nos inspira la nostalgia de la fiesta primitiva y nuestro arte intenta resucitarla. Es precisamente en estas formas de arte colectivo donde la distancia del crear y del percibir se oculta, y la creatividad se generaliza. (Dufrenne, 1967, p. 448). En estas obras de carácter colectivo, el público participa y se divierte, pero para ello el artista debe renunciar a "sus vanos privilegios" y se pide que la obra abandone el museo, que descienda a la calle, que sea acontecimiento, un hecho de todos; hecho de la ciudad..., hecho de los insurrectos en las barricadas. 


\subsubsection{Teorías para el desarrollo de lo colectivo en el arte}

Cercano a Dufrenne, con quien co-dirigió la colección "Esthétique" "de las Editions 10/18 en los años 70, Revault d'Allonnes, heredero y difusor de la Escuela de Francfurt, en especial de Adorno, proclamó el valor de la aportación de lo colectivo al arte. "Ya hace tiempo el tipo humano del poeta, del pintor, del compositor individuales, espléndidamente solitarios, ha dejado de corresponder a la realidad" (Revault D'Allones, 1977, p.271).

El arte contemporáneo se basa al fin, en muchas de sus obras, en lo que podemos llamar el genio de varios ..., o el genio colectivo, en las formas parcial o enteramente espontaneas, como cierto tipo de teatro, de coreografía, de músicas colectivas improvisadas, el happening, la fiesta, etc.

El teórico francés, profundizó en el estudio de un arte colectivo como es el de la Rebétika arte musical vocal y bailado, (Revault D'Allones, 1977, pp.141-173) desarrollado en la Grecia urbana periférica. Arte reivindicativo, de protesta y de oposición que ha ejercido funciones sociales destacables, representante y expresiva de una identidad cultural de grupo, forjada en los márgenes de la sociedad más desposeída, que ha soportado periodos de represión constantes (Revault D’Allones, 1977, p.173). El estudio caracteriza en parte el arte colaborativo, en su momento.

A la Escuela de Frankfurt pertenecieron teóricos de un nivel extraordinario cuya impronta no ha desaparecido. Es el caso de Walter Benjamin con su teoría de la pérdida del aura del arte en la era de la reproductibilidad. Ese texto abrió nuevas perspectivas a la percepción artística para toda la sociedad. Benjamin ejerció una gran influencia sobre Adorno, sin embargo, su visión positiva sería transformada en crítica en su discípulo y amigo. La teoría de Theodor Adorno ha sido una de las más influyentes del siglo XX. En ella se establece que todo arte tiene esencia social y permanecerá incomprendido y falto de su verdad total, si no se comprende esa esencia. “El arte todo está compuesto de algo formado en sí mismo por su propia lógica y consecuencia y también por la conexión entre espíritu y sociedad" (Adorno,1980, p.456). Sin embargo, la eficacia de la obra está en su propia configuración. Todo proceso de constitución de una obra tiene eficacia social como modelo de praxis posible en la que se constituye una especie de sujeto colectivo (Adorno,1980, p.316).

Ahora bien, los análisis externos a la obra que solo pretenden medir su eficacia social, ignorando las tensiones entre contenido y eficacia, no respetan la verdad total de la obra; tampoco lo hacen los que la aíslan, es preciso tener en cuenta "la hora histórica en que aparecen "(Adorno, 1980, p.316). Las obras de arte tienen su eficacia práctica en una masificación de la conciencia a penas concretable y no en que se pongan a arengar" (Adorno, 1980, p.317).

De cara a nuestro ámbito de estudio tienen especial relevancia las reflexiones de Adorno y Horkeimer, sobre las industrias culturales (1944). Sus críticas se refieren a los films, la radio, los periódicos (los semanarios) y la publicidad. Es decir, los años 40 contemplaban un ámbito mucho más restringido de las Industrias culturales en relación con el expandido que vivimos en la actualidad. Su concepción de las industrias culturales como negocio y su señalamiento del dominio de la taquilla (tiranía actual de las taquillas) fueron aurorales (Horkheimer y Adorno 1970, p. 165) "Adaptación consciente a las necesidades del público calculadas según las cifras de boletería” (taquilla) (Horkheimer y Adorno, 1970, p.165). 
En el campo artístico, el estilo ha sido siempre negado en las grandes obras, los grandes artistas han preferido atenerse menos al estilo que a "la lógica del objeto" (Horkheimer y Adorno, 1970, p.158). El estilo de la gran obra de arte se ha visto siempre negado, la obra mediocre ha preferido siempre semejarse a las otras, se ha contentado con el sustituto de la identidad. La industria cultural, entendida como "la industria de la diversión" (Horkheimer y Adorno, 1970, p.164), absolutiza la imitación. Reducida a puro estilo, traiciona el secreto de este, o sea, declara su obediencia a la jerarquía social (Horkheimer y Adorno, 1970, p.158). Consagra la reiteración, fundamentalmente respecto a los estereotipos (Horkheimer y Adorno, 1970, p.164) y consiste en "la exclusión de lo nuevo" (Horkheimer y Adorno, 1970, p.162) El imperio del consumo descarta como riesgo inútil lo que aún no ha sido experimentado (Horkheimer y Adorno, 1970, p.162).

\subsubsection{Nuevas aportaciones teóricas en la segunda mitad del siglo}

Heredero de la primera Escuela de Frankfurt, Jürgen Habermas con sus elaboraciones teóricas, eleva con fuerza el concepto de Sociedad Civil al calor del abordaje de otras nociones como la de Espacio público, Esfera pública y Opinión Pública.

Establece la materialización del concepto de esfera pública, con la aparición del Estado Moderno, así como el surgimiento de la esfera que le hace frente, la sociedad civil. (Habermas, 2019, p.16). Ambos conceptos van de la mano. La Opinión pública es la expresión de la sociedad civil, "un freno que" ...se "puede oponer ... a la acción del gobierno y de la administración, pero que puede ser circunscrito en función de resultados y conclusiones forjadas por sondeos", y corre el riesgo de "ser manipulado por los medios apropiados" (Habermas, 2019, p.253).

El espacio público de Habermas hace ver la importancia de la sociedad civil compuesta de individuos independientes muy heterogéneos, que tiende a contrabalancear el poder de los que controlan al Estado (Paquot, 2015, p.22). Subraya la importancia de una sociedad civil que ya le había otorgado Marx y a la que reconocía una relativa autonomía (Paquot, 2015, p.23). Su concepción del Espacio Público quedaría configurada como: lugar de encuentro y visibilidad entre sujetos libres e iguales que razonan y argumentan espontáneamente, liberados de cualquier presión del poder, en un debate que busca el entendimiento mutuo. Internet ha ampliado la noción de espacio público, aunque en realidad deberíamos decir que internet ha coronado y llevado al mayor nivel la idea de intervención de los individuos comunes.

Esfera pública es el ámbito configurado por los espacios públicos de debate y cuestionamiento que posibilita el surgimiento de la opinión pública así como de las organizaciones cívicas, de colectivos emanados de la sociedad que elevan su voz crítica frente al poder instituido de una democracia que no reconoce la sociedad constituida absolutamente por "pares", lo que si podría lograrse en el seno de una democracia deliberativa, es decir, en un modelo político reordenado por un procedimiento colectivo en donde la toma de decisiones se realiza mediante la participación activa de todos los potencialmente afectados, a través de la deliberación, es decir, por medio de la argumentación y discusión de propuestas discutidas públicamente.

De esta manera la Esfera Pública queda configurada en Habermas, tal y como Velasco Arroyo (2014, p.170) lo fija, por aquellos "espacios de espontaneidad social libres de interferencias estatales, de las regulaciones del mercado y de los medios de comunicación poderosos". Son espacios de discusión y deliberación de donde surge la opinión pública en su fase informal, así 
como las organizaciones cívicas y, en general, todo aquello que desde fuera cuestiona, evalúa críticamente e influye en la política." En términos normativos, la esfera pública (Öffentlichkeit) (Habermas, 2019, p.13) puede entenderse como aquel espacio de encuentro entre sujetos libres e iguales que argumentan y razonan en un proceso discursivo" (Diskurs: de controversia entre varias personas) abierto dirigido al mutuo entendimiento (Velasco Arrollo, p. 170 y p. 214).

Habermas entiende que esos espacios y esa esfera pública podía desarrollarse en el ámbito de un tipo determinado de la democracia: en la democracia deliberativa, con "resolución racional de conflictos políticos por medio de prácticas argumentativas o discursivas en diferentes espacios públicos". Se trata pues, de elevar a rango institucional la teoría de la argumentación pública, pero cabe preguntarse ¿para qué, o dónde? La respuesta es: "en el proceso legislativo". Es decir, en el más alto nivel político. El eco de su propuesta nos envuelve.

Por su parte, la renovación marxista de Lucien Goldmann, desde un "estructuralismo genético", ya en la segunda mitad del siglo XX, retoma la noción de conciencia colectiva (designación de un conjunto de conciencias individuales y sus relaciones mútuas), que no se ubica al margen de estas (Marx y Piaget) subrayando que la existencia de todo grupo social constituye un proceso de equilibrio entre un sujeto colectivo y un medio social natural. Su atención al mundo artístico se centra en el mundo literario, y concretamente en la novela, donde establece la relación entre la práctica político-económica y la práctica cultural en cuanto la forma novelada supone la trasposición, en el plano literario, de la vida cotidiana (Goldmann, 1975).

Especial interés tienen los textos de Paul Ardenne dedicados al Arte Contextual; caracteriza al arte así denominado como manera de hacer por medio del cual "el artista se proyecta ahora en el corazón del mundo y de los suyos, posicionado para un trabajo que compromete prácticas de intersubjetividad, de reparto y de creación colectiva" (Ardenne, 2002, p.28). La pulsión participativa que este tipo de arte supone, "requiere compromisos puntuales, políticos o éticos" (Ardenne, 2002, p.28). La acción artística, como "toda experiencia tiene algo de provocación y viene a provocar lo que ha sedimentado el orden establecido", perturba lo que el orden de las cosas manda no trastocar, por tradición, pereza o estrategia. (Ardenne, 2002, p.32).

Gran relevancia, en el caso del arte contextual, tiene el Process Art, en el que "la acción artística importa por lo menos tanto como el resultado obtenido". Apropiarse la realidad, viene a ser entonces activar en ella un "proceso". El arte contextual es "por morfología "un arte del acontecimiento (Ardenne, 2002, p.35). Un arte insumiso a los poderes públicos:

Activista, volátil, el arte público no programado suscita la aprobación o la ira de los poderes públicos, que dejan hacer o prohíben según la relación de fuerzas del momento o que también recuperan este arte de insumisión a buen precio, que les permite hacer valer en sitio público su gusto por lo subversivo. (Ardenne, 2002, p.56)

Este arte es un arte participativo y "el arte participativo es muestra de la solicitación, busca de manera abierta y a menudo espectacular la implicación del espectador" (Ardenne, 2002, p.122); es una realización "donde la intersubjetividad se revela como mecanismo de creación" (ibidem).

Señala el "otrismo" (por oposición al autismo) (Ardenne, 2002, p.123), como la naturaleza de las obras participativas, obras hechas "para el espectador y con él" (ibidem) En estos casos, el artista aparece preocupado por la cuestión de la "acción grupal" (Ardenne, 2002, p.124), ya que la obra 
"otrista" tiene por vocación suscitar un "estar juntos". A la espera de una mayor solidaridad social (al menos localmente), apostando por la posibilidad de una intersubjetividad (realizada al menos de manera pasajera)" (Ardenne, 2002, p.124). Esta manera de hacer, participativa, refuerza la democracia vivida, "de la que es, probablemente, el más significativo hijo simbólico" (Ardenne, 2002, p.124).

Enfocándonos ahora hacia la temática de la ciudad, la aportación de una figura como Lefebvre es fundamental. En la línea marxista, atemperada con el paso del tiempo, Henri Lefebvre, uno de los principales inspiradores del mayo del 68 , se interesa por la crítica de la vida cotidiana y por los problemas que acucian a la ciudad contemporánea. Ciudad a la que teniendo derecho todos los individuos de una sociedad, muchos quedan excluidos, al margen de toda decisión que les afecte. Detecta y alerta sobre una falta de participación, una falta de intervención por parte de los interesados.

Para que esta situación se pueda revertir se precisa una transformación de la sociedad, lo que supone la posesión y la gestión colectiva del espacio, por intervención permanente de los concernidos, "con sus múltiples intereses, diversos e incluso contradictorios" (Lefebvre, 2000, p.484) y pasa por la negación activa, teórica y práctica de la situación actual, a través del contraproyecto, el contra-plan. Así pues, por la intervención activa y masiva de los "interesados" (Lefebvre, 2000, p.481) con el fin de poder alcanzar una "vida nueva (...) generalmente comunitaria" (Lefebvre, 2000, p.437). Hasta ahora esto no se ha dado, quizá a falta de una "invención morfológica” (Lefebvre, 2000, p.437).

Lo urbano quedaría así definido "como lugar de expresión de los conflictos" (Lefebvre, 1983, p.181) "La incompatibilidad entre lo estatal y lo urbano es radical" (Lefebvre, 1983, p.184). Por ello, "lo urbano, solo puede constituirse y servir "al habitar" derribando el orden estatal y la estrategia que organiza de manera opresora y homogeneizante el espacio. (Lefebvre, 1983, p.185). Lo que se modela es un espacio político (Lefebvre, 1983, p.185).

Entre los derechos que definen una civilización como son "el derecho al trabajo, a la instrucción, a la educación, a la salud, al alojamiento, a las aficiones, a la vida, figura el derecho a la ciudad" (Lefebvre, 2016, p133). Si estos derechos, que aún se encuentran en formación, se pusieran en práctica cambiaría la realidad, pero esto no llegará a materializarse si la clase obrera se calla (Lefebvre, 2016, p.113). Lefebvre insiste una y otra vez ¿por qué este silencio de los usuarios?” (Lefebvre, 2016, p. 186).

Contestación a la demanda de acción la encontramos en William Bunge, con él penetramos en el ámbito geográfico urbanístico como geógrafo que formó parte de la llamada geografía radical, centrada en el análisis del espacio social. Dedicó sus esfuerzos a estimular las posibilidades de reaccionar contra las desigualdades sociales. Es decir, a aplicar los conocimientos geográficos para producir mejoras en el campo social.

La Expedición Geográfica a Detroit fue un ejemplo del deseo de Bunge de poner sus conocimientos y herramientas metodológicas al servicio de una comunidad con mayoría de personas de color, en riesgo de convertirse en ghetto. Recabando información de entre aquellos que eran sus propios vecinos y compartiendo sus conocimientos. Bunge vivió y trabajó entre 1969 y 1970 en colaboración con los habitantes del barrio Fitzgerald de Detroit, ya lo había hecho en Chicago y lo hará más tarde en Toronto. Reivindicando el valor de los trabajos de campo, e introduciendo "como elemento de lucha, el control del propio espacio, las formas 
en que las distintas comunidades pueden ejercer el control de sus distintos espacios". (Gómez Mendoza, 1988, p.159). Sus acciones se nos presentan como materialización puntual del clamor de Lefebvre.

El requerimiento de Lefebvre se prolonga y acentúa a través de David Harvey. Como seguidor de Lefebvre, reivindica que el derecho a la ciudad es ...pretender un poder de configuración (façonnement) fundamental y radical sobre los procesos de urbanización, es decir sobre las maneras en que nuestras ciudades son transformadas sin cesar (Harvey, 2011, p.9). Y profundizar en la "construcción de un amplio movimiento social para que los desposeídos puedan retomar el control de esta ciudad de la cual son excluidos", pero también para que puedan "instituirse nuevos modos de control de superavit de capital que forma el proceso de urbanización" (Harvey, 2011, p.33).

Todos los implicados en la producción de la ciudad "disponen de un derecho colectivo no solamente a lo que ellos producen, sino también a decidir lo que debe ser producido, donde y cómo" (Harvey, 2011, p.92). El derecho a la ciudad es "un derecho situado". "Incluye no solamente a los trabajadores del edificio", si no también todos los que intervienen en el desarrollo de la vida cotidiana: "Ios que prodigan los cuidados y los enseñantes, los que trabajan en las alcantarillas..." (Harvey, 2011, p.93)

Harvey, siguiendo la expresión desiderativa de Lefebvre, adopta un tono más imperativo al exigir una acción eficaz: Los productores urbanos deben levantarse y reclamar el derecho a la ciudad que ellos producen colectivamente.

\subsubsection{Dos miradas específicas sobre el beneficio de lo colaborativo}

El término colaborativo fue acuñado en 2006 por Yochai Benkler. Ahora bien, los planteamientos se entienden enraizados en la defensa de los bienes comunes y pasan por la teoría de Elinor Awan, más conocida como Elinor Ostrom, por el apellido de su marido. Ella demostró al mundo que la gestión por parte de los usuarios de recursos comunes resultaba, en la mayoría de los casos, mucho más positivo de lo que se decía y que a su vez esos usuarios de bienes comunes desarrollan con frecuencia sofisticados mecanismos de toma de decisiones, así como de resolución de conflictos de intereses, con resultados positivos. (Argumentación del Comité para la concesión del Nobel, Premios 2009, en PUCHE, 2019, p.7). Como es bien sabido, por sus aportaciones en nuestro campo, fue reconocida con el Premio Nobel de Economía en 2009.

Una línea opuesta que tiene antecedentes históricos (Aristóteles, Hobbes, Foster Lloyd y Scott Gordon, entre otros), parece culminar en los planteamientos que se amparan en torno al artículo de G. Hardin (1968) titulado "La tragedia de los comunes". Prácticamente en todos aparece el argumento del descuido o menor cuidado de los individuos hacia lo común, porque los hombres tienden a mirar por su propio bien.

A ellas dio contestación Ostrom con sus teorías de valoración de la gestión comunal de bienes (1990), en una tercera vía, opuesta tanto a la gestión del Estado, como la del mercado. Ni Estado, ni Mercado (una supuesta adaptación de la formula anarquista: Ni Dios, Ni Estado). Lo que se observa en el mundo es que ni el Estado ni el Mercado han logrado con éxito que los individuos mantengan un uso productivo, de largo plazo, de los sistemas de recursos naturales. Además, distintas comunidades de individuos han confiado en instituciones que no se parecen 
ni al Estado ni al Mercado para regular algunos sistemas de recursos con grados razonables de éxito durante largos periodos (Ostrom, pp. 35 y 36; citado en Puche, 2019, p. 24).

Elinor Awan (Ostrom) señala una serie de condiciones para el gobierno y uso de los bienes comunes (RUC: recursos de uso común) con el fin de lograr instituciones de larga duración, algunas de ellas de gran interés: tras determinar los límites de los recursos, así como los individuos o grupos con derecho a beneficiarse de los RUC; se debe buscar la coherencia entre las reglas de apropiación y provisión con las condiciones locales; Igualmente, determinar las condiciones de la participación en arreglos de elección colectiva, así como en los aspectos relativos a la supervisión en las condiciones del RUC y quienes la ejercen; importante, la elaboración de las sanciones graduadas para quienes violan las reglas operativas que rigen en los RUC; y la adopción de mecanismos para la resolución de conflictos; finalmente, obtener el reconocimiento por parte de las autoridades gubernamentales de las instituciones creadas por los RUC. En el caso de Recursos de Uso Común que formen parte de sistemas más amplios, se ejercerá supervisión de todas las actividades (apropiación, provisión, supervisión aplicación de normas, resolución de conflictos y gestión,) que se organicen en niveles múltiples de entidades incrustadas. (Ostrom, p. 165; citado en Puche, 2019, pp.51-53).

Siguiendo a Ostrom: Benkler define lo colaborativo como "modalidad de organización productiva ...basada en recursos y productos compartidos entre individuos ...que cooperan sin depender de directrices mercantiles o de órdenes jerárquicas". A esta nueva modalidad la denominó "producción entre iguales basada en el procomún" (Benkler, p. 98). El procomún "alude a una forma institucional de estructurar el derecho de acceso, uso y control de los recursos". En definitiva, se está refiriendo a un "sistema de producción, distribución y consumo de bienes de información que se caracteriza por acciones individuales descentralizadas, ejecutadas a través de medios ampliamente distribuidos y ajenos al mercado y a sus estrategias» (Benkler, p.97). La aportación de Benkler se ha centrado en el caso de los programadores de software libre que actúan sin seguir las directrices generadas por modelos mercantiles... que se apartan de la economía de la información industrial mediante la mejora de la eficacia de la producción no mercantil en general (Benkler, p.98)

Ahora bien, señala como no todas las iniciativas productivas basadas en el procomún reúnen los requisitos que definen la producción entre iguales. "Alude a sistemas de producción que dependen de una acción individual autodeterminada y descentralizada y no asignada jerárquicamente". Son prácticas de acción colectiva muy eficaces, descentralizadas, que "no se apoyan para su coordinación ni en el sistema de precios ni en una estructura directiva. Surge así una "plataforma para nuevos mecanismos que permiten que agentes muy dispersos adopten estrategias cooperativas radicalmente descentralizadas en lugar de recurrir a alegaciones privativas y contractuales para establecer precios o imponer órdenes directivas (Benkler, p.101).

La quintaesencia de esta producción entre iguales basada en el procomún ha sido el software libre, o de código abierto, basado en el esfuerzo compartido (Ibidem) de muchas personas que comparten sus respectivas contribuciones sin que ninguna persona o entidad reclame derechos exclusivos sobre los componentes que ha aportado sobre el conjunto resultante. Para evitar ese peligro, "los participantes suelen mantener el copyright de sus contribuciones, pero lo ceden a los demás ...según un modelo que combina un permiso universal de uso con restricciones que hacen difícil, si no imposible, que ningún participante o tercera parte se apropie del proyecto. Este modelo de Licencia es "la innovación institucional más importante del movimiento de 
software libre. Y su ejemplo fundamental es la Licencia Pública General (GPL) por sus siglas en inglés) de GNU "que exige se respete las mismas condiciones de libertad del programa original," sea quien sea quien utilice, modifique o distribuya un programa bajo su cobertura. (Ibidem).

Hemos ido caracterizando paulatinamente las diferentes nociones del mundo colaborativo a través de una selección de fuentes sin pretensión de agotarlas. La noción de unión fortalecida por Marx, Engels y Bakunin, la de apoyo mutuo por Kropotkin, la oposición a la autoridad con Bakunin el reconocimiento del otro con Husserl y la apertura al otro como yo con MerleauPonty desde una opción de izquierdas sin etiqueta (tercera vía), el impulso del arte colectivo desde Dufrenne y la caracterización de arte colectivo por Revault D’Allones, determinación de los espacios públicos y la de esfera pública, como ámbitos de desarrollo del arte de sujetos libres e iguales que trabajan por el entendimiento, que tienen en otros teóricos su aplicación directa al mundo del arte como el caso de Ardenne, como en otros muchos. La aplicación al mundo de la ciudad como derecho de todos en el pensamiento de Lefebvre y Harvey, el derecho a integrar las instancias decisorias y su plasmación práctica en el quehacer de Bunge (quartier Fitzgerald), todo ello, ha ido configurando el caldo de cultivo que se va a ir concretando con las teorías de Elinor Ostrom y Benkler, fundamentalmente, que constituyen el núcleo de la teoría relativa al universo colaborativo que se sitúa al margen del mercado

\section{DISTRITOS CULTURALES}

En primer lugar, constituyen ámbitos en los que se desarrollan actividades culturales. Surgen de un clima económico capitalista cuyo objetivo es el rendimiento creciente del dinero, aunque específicamente se trata de mejorar las condiciones económicas disminuidas, a causa de una crisis provocada por la búsqueda de un mejor rendimiento económico industrial (emigración de industrias a sedes más baratas). Es decir, que vienen a solucionar un problema generado por el propio mantenimiento y/o crecimiento industrial. La dirección que ellos adopten en su desarrollo depende de los gestores. La cultura erigida como motor económico podría tener aspectos beneficiosos para la propia cultura, no es de partida un aspecto negativo, depende de la gestión, de la orientación político-social de la gestión que se implante. Históricamente la crítica a los distritos culturales ha provenido, en general, de la crítica al contenido y a la gestión de las filières que constituyen las Industrias culturales desarrolladas en ellos. La interpretación de la gestión de la cultura desde la taquilla ha sido el gran mal para la consideración de los Distritos, De ahí la crítica de Adorno y Horkheimer que, siendo una crítica a las Industrias culturales, no a los Distritos, que no existían como tal, sigue estando vigente aplicado a los Distritos como ámbito de desarrollo de las Industrias culturales en el seno del mercado.

Con el paso del tiempo, los distritos culturales fueron siendo asumidos por las Distritos Creativos a tal nivel que en la actualidad el sector digital, experimenta un auge ampliado a las llamadas industrias de contenidos digitales que se expanden de manera imparable y "con la consecuente repercusión en la pérdida de espacio de las Industrias Culturales" originarias "en función de un proceso de repliegue que ya se inició con la asunción de las Industrias Creativas y que en estos momentos siguen creciendo, solicitadas por la necesidad de las circunstancias, mientras pierden peso de forma significativa las industrias basadas en el patrimonio y el resto de las llamadas Industrias Culturales que están sufriendo un golpe de herida mortal" (Aumente, 2020, 606). Actualmente el sector digital, incluidas las industrias de contenidos, va conquistando espacios nuevamente urbanizados o reactivados por colectivos de artistas que repoblaban antiguas zonas industriales que hoy de nuevo se regentrifican por especialistas en las TICs, expulsando, 
incluso a los artistas. No obstante, esto podría conllevar, en algunos casos, la incorporación de colectivos artísticos digitales procomunes que trabajan en red, aunque suelen ser más frecuentes todavía en squats marginales, aunque cada vez más integrados.

Las teorías sobre los Distritos culturales han ido evolucionando, como lo han hecho los propios distritos. Analizando los escritos y las propuestas de teóricos como Sacco y Ferilli, que han ahondado en el tema durante años, encontramos unas propuestas que pretenden acortar diferencias con los presupuestos colaborativos, sin, por supuesto, asumirlos plenamente. Huyendo de la pretensión de agotar las posibles menciones, puesto que además he trabajado durante años el tema de los distritos culturales, me he centrado exclusivamente en aquellos aspectos que tienen que ver directamente con el objetivo marcado. Veamos pues, esas referencias que podrían ser esclarecedoras.

Sacco y Ferilli abogan por unos distritos culturales evolucionados: El Distrito Cultural por ellos definido "amplía las consideraciones de referencia, de manera señalada en lo que se refiere a la conservación del Patrimonio de Bienes Muebles, así como lo relativo al paisaje cultural y los jardines, pero fundamentalmente en lo concerniente a la concertación con la sociedad" (Aumente, 2020, p.606), el desarrollo del proyecto ha de estar refrendado por el acuerdo con la sociedad. La presencia cooperadora de la sociedad civil (asociaciones culturales de todo tipo, incluyendo las preocupadas por el desarrollo sostenible y los presupuestos ecológicos), es requisito imprescindible.

Presentan el distrito cultural sistémico como: "Centro sinérgico del conjunto de la economía local basada en la innovación" (Ferilli y Sacco, s.a. p.47). Para el desarrollo de estos centros sinérgicos "la participación e implicación de la sociedad es imprescindible. Comparadas con la ciencia y la tecnología, la cultura y la creatividad son más fácil y rápidamente accesibles y poseen capacidades constructivas (potenciales) del conjunto de la comunidad local, asegurando así su participación e implicación, algo menos previsible en el ámbito científico y tecnológico" (Ferilli y Sacco, s.a., p.47). No es su única referencia a este aspecto, en sus textos se insiste una y otra vez en la importancia de la implicación y participación de los concernidos, cabría, en este punto, preguntarse si es posible detectar los ecos de Lefebvre.

Para la implementación de los proyectos de distritos culturales se recomienda buscar "lugares donde existan iniciativas empresariales autónomas y un alto nivel de vida comunitaria y de participación" (Ferilli y Sacco, s.a. p.48). Es importante esa participación de los ciudadanos y de la comunidad local y puede estimularse "mediante acciones y políticas proactivas" (Ferilli y Sacco, s.a. p.62). Antes que nada, y este aspecto tiene especial relevancia, es necesario un "pacto social de desarrollo inclusivo y creible" (Ferilli y Sacco, s.a. p.48).

Para estos tres teóricos italianos, "a la sinergia cultural, se añade la económica y fundamentalmente la social "(Sacco; Ferilli y Tavano, 2012, p.109), por lo que proponen doce acciones políticas entre las que, desde nuestra óptica, es preciso destacar las orientadas al desarrollo de la comunidad local afectada:

-Presentar una oferta cultural de calidad: realizar proyectos culturales. Involucrar a los actores locales que, al tiempo, ofrezca interés para actores externos manteniendo un alto nivel de vinculación a la comunidad local que no debe sentirse excluida de la actividad cultural, ni verla distante e inalcanzable (Sacco, Ferilli y Tavano, 2012, p.104). Influencia de Florida en cuanto a la capacidad de atracción externa, pero con sensibilidad hacia lo local, propiciando fuerte 
inclusión (Ya Marshal mencionaba la importancia de la conexión con la comunidad cercana al distrito industrial).

-Capacitar y formar a la comunidad local. Debe permitir, a los individuos que conforman la comunidad, la superación de la dificultad que conlleva el acercamiento a realidades diferentes y complejas (Sacco, Ferilli y Tavano, 2012, p.104). La capacitación y la formación de la comunidad local (cfl) son prioridades consideradas estratégicas a corto plazo (Sacco y Ferilli, s.a. p.60). Influencia de Sen y sensibilidad social.

-Suministrar los instrumentos para modificar la lógica que guía las relaciones sociales de la comunidad local de una manera prosocial (ligada a la capabilities de Sen) manteniendo alta el deseo de cambio social. (Sacco, Ferilli y Tavano, 2012, p.105).

Se enumeran igualmente otras acciones que tienen que ver con el desarrollo del talento local y el mantenimiento de la identidad cultural (Ibidem), pero, de nuevo, se insiste en la importancia de la:

\begin{abstract}
Participación de los ciudadanos y de la comunidad local: la dimensión de la participación es fundamental para el éxito de cualquier iniciativa. En particular la de la comunidad local que, gracias también y sobre todo al proceso de capacitación, está en posición de apreciar la propiedad y de traducir de los lenguajes culturales en el ámbito de sus prácticas culturales y de su propio sistema de valores (Sacco, Ferilli y Tavano, 2012, p. 105)
\end{abstract}

Y desde el punto de vista de la gobernanza:

Asegurar una gobernanza local de calidad. Afianzar la capacidad de coordinación de las acciones de los diversos actores y de los concernidos del proceso de desarrollo, lo que no quiere decir que la iniciativa deba partir de la administración pública, es suficiente que esta desarrolle un papel de "facilitador" de acciones innovadoras y de proyectos y que sea mediador entre los diversos intereses en juego. (Sacco, Ferilli y Tavano, 2012, p.105).

Por supuesto, no se trata de planteamientos que sigan enfoques colaborativos, tampoco estas propuestas parecen situarse en posiciones absolutamente irreconciliables. En relación con este último punto, aparte de que este planteamiento da carta de naturaleza a los proyectos de Distritos Culturales no surgidos desde arriba (iniciativa de administración pública), sino desde abajo, ¿podría aceptarse la posibilidad de afirmar que aquí se deslizan acercamientos, en mixtificación ecléctica con otros de diversa etiología, a las posiciones de Proud'hom recogidas por Bakunin? El Estado, la Administración, como simple facilitador y mediador de las iniciativas innovadoras surgidas de la sociedad... Un cierto viraje, algunos acercamientos, por supuesto muy atemperados, parecen detectarse. Lluvia fina. 


\section{CONCLUSIONES}

En primer lugar, desde una perspectiva general, en los dos polos objeto de investigación, se observan planteamientos diferentes, distintas trayectorias, distintos objetivos, no obstante, no tiene por qué tratarse de dos ámbitos absolutamente irreconciliables.

Una mirada actual más detenida, dejará ver que, sin embargo, ciertos aspectos del Distrito Cultural Sistémico, bajo la influencia de las teorías de Sen, pero con una conciencia social más sensibilizada, requieren una mayor implicación de la sociedad y una mayor participación. Ahora bien, implicación y participación, son conceptos cercanos a cooperación, pero no son exactamente lo mismo que colaborativismo. La introducción de esos modos de actuación puede ser paulatina en una actuación constante y progresiva. Si bien es cierto que no suele aparecer la impronta colaborativa en el diseño de la mayor parte de los distritos culturales, puede implantarse, sin embargo, a posteriori, en su desarrollo.

Dado ya un determinado distrito, esa participación, gestionada desde una posición más colaborativa puede conseguir la aprobación de proyectos comunes para trabajar en conjunto. Un ejemplo de ello podría ser la aprobación de actividades a realizar por colectivos que trabajen desde la pluralidad, buscando no ya la aprobación del público, y la rentabilidad económica, sino la colaboración -tanto en la concepción como en la realización del proyecto-, de los integrantes de la sociedad local, trabajando todos en plano de igualdad y con el objetivo puesto en la estimación personal y social de su trabajo.

En un segundo nivel, la lógica colaborativa con la mirada puesta en un futuro, lo más cercano posible, debe aspirar a que la capacidad decisoria de la comunidad social de base obtenga espacios, no solo de debate, si no de decisión sobre la instauración y el desarrollo de Distritos Culturales desde el comienzo de los proyectos. Las transformaciones de los espacios urbanos vienen de la mano de las transformaciones sociales y a la inversa. ¿Utopía del trabajo en común?, el objetivo está puesto en que, los ahora limitados ensayos, se conviertan en escenario habitual en breve.

Un tercer aspecto es necesario mencionar, la absorción del campo de los Distritos culturales y de los barrios artísticos por las industrias creativas. Esta reflexión insta a mantener y redoblar esfuerzos desde lo colaborativo en ese campo, donde ya existe, a nivel de las redes, un comportamiento brillante (Linux).

La posible conciliación solo podría lograrse al invertir la dinámica: del Distrito CulturalComunidad Local, a Comunidad Local-Distrito emanado de la acción conjunta y colaborativa, a espaldas del mercado (en línea con el crowdfunding o similares), de los miembros de dicha comunidad en abierta conexión y sinergia con el mundo.

\section{APOYOS}

Proyecto Arte, Arquitectura y Patrimonio en los procesos de construcción de la imagen de los nuevos enclaves culturales (del Distrito al Territorio). Ministerio de Ciencia, Innovación y Universidades, Programa Generación de Conocimiento (ref. PGC2018-094351-B-C43). 


\section{REFERENCIAS}

Adorno, Th. (1980) Teoría Estética. Madrid, España: Taurus.

Althusser, L. y Balibar, E. (2019). Para leer el Capital. México: Siglo XXI.

Ardenne, P. (2002) Un arte contextual. Creación artística en medio urbano, en situación, de intervención, de participación. Murcia, España: Cendeac.

Aumente, P. (2020) Distritos Culturales. Cuestiones de concepto a revisión. En Chaves, M. A. E Ed. Visiones Urbanas. Madrid, España: Grupo de investigación Arte, Arquitectura y Comunicación en la ciudad contemporánea Universidad Complutense de Madrid. (UCM). Tesis de doctorado (1985). La Estética y la Teoría del Arte en la Escuela Fenomenológica Francesa: Mikel Dufrenne. Madrid España: Universidad Autónoma de Madrid. (Inédita).

Bakunin, M.A. (1894) Dios y el Estado Feedbooks. Reedic. en Ebook. Amazon. Kindle. Estatismo y anarquía. (1873). Feedbooks. E- book. Amazon. Ed. Kindle. Federalismo, socialismo y antiteologismo. 1868. E- book. Amazon Ed. Kindle.

Benkler, Y. (2015). La Riqueza de la Redes. Cómo la producción social Transforma los Mercados y la Libertad. Barcelona: Icaria. Ed. digital libre.

Dolgoff, S. (2017). La anarquía según Bakunin. Ariel. Amazon. Ed. Kindle.

Dufrenne, M. (1967). Phénoménologie de l'experience esthétique. París, France: P.U.F. (1981). L'inventaire des a priori recherche de l'originaire. Paris, France: Christian Bourgois. (1974). Art et Politique. Paris. France: Union Générale d’Editions.

Ferilli, G. y Sacco, P.L, (s.a.). Los Distritos culturales sistémicos. Sevilla, España: Editorial Doble J.

Goldmann, L. (1975). Para una sociología de la novela. Madrid, España: Ayuso.

Gómez Mendoza, J. (1988). Las Expediciones geográficas radicales a los paisajes ocultos de la America urbana, en Gómez Mendoza, J. y Ortega Cantero (1988). N. Viajeros y paisajes. 
Garnham, N. (2011). De las industrias culturales a las creativas. Análisis de las implicaciones en el Reino Unido. En Bustamante Ramírez, E. (Coord) Las industrias creativas: amenazas sobre la cultura digital. pp. 21-48.

Habermas, J. (1992). L'Espace Public. Archéologie de la publicité comme dimensión constitutive de la société bourgeoise. Millau, France: Payot et Rivages. (Reimpr de 2019).

Harvey, D. (2011). Le capitalisme contre le droit à la ville. Néolibéralisme, urbanisation, résistances. Paris. France: Éditions Amsterdam.

Horkheirmer, M. y Adorno, T. (1970) la Industria cultural. Iluminismo como mistificación de masas. En Dialéctica del iluminismo. B. Aires: B. Aires Sur.

Husserl, (1982). La idea de la fenomenología. México. México: Fondo de Cultura Económica.

Kessler. Revista de Divulgación e Investigación en Ciencias Naturales. 1/4/2005. Molinero, A. Titanes en la sombra: Kessler y la ley de la ayuda mutua. Recuperado de https://gonmoal.blogs. uv.es/2015/04/01/115246462280/

Kropotkin. P. (2016) El Apoyo Mutuo. Un factor de evolución. Logroño, España: Pepitas de Calabaza.

Lefebvre. (2000). La producción de l'espace. Barcelona. España: Antrhopos. (2016) Le droit à la ville. Paris. France: Anthropos, 3a ed. 2a reipr. (1983.) La revolución urbana. Madrid, España: Alianza Editorial. 4a ed.

López Cuenca, A. (2016) Los comunes digitales. Nuevas ecologías del trabajo artístico. México. México: Secretaría de Cultura. Centro Cultura. Recuperado de https://editorial. centroculturadigital.mx/libro/los-comunes-digitales-nuevasecologias-del-trabajo-artistico

Malthus, T. Primer ensayo sobre la población, (s.a.) Recuperado de https://museo-etnografico.com/pdf/ puntodefuga/171128malthus.pdf Ejemplar de ediciones Altaya.

Marcuse, H. (1981), Eros y Civilización. Barcelona, España: Ariel. Marx, K. y Engels, F. (2015). El Manifiesto Comunista. e-artnow. Ed. Kindle. El Capital II. Edic. (1870). Dominio Público. Ed. Kindle. 
Merleau-Ponty, M. (1975). Fenomenología de la percepción. Barcelona. España: Península. (1971) Existence et dialectique, Textes Choisis. France: PUF. (1977) Sens et non sens, versión española: Sentido y sinsentido. Barcelona, España: Península. (1955) Humanismo y Terror. Recuperado de https://www.uv.mx/ tipmal/files/2016/10/M-MERLEAU-PONTY-HUMANISMO-YTERROR.pdf

Molinero A. G. (2015). Titanes en sombra: Kessler y la ley de la ayuda mutua. Revista de divulgación e Investigación en Ciencias Naturales. La libertad de divulgar. https://gonmoal.blogs. uv.es/2015/04/01/115246462280/

O'Connor, J. (2011). Las industrias creativas y culturales: una historia crítica. Ekonomiaz, 78, 3. cuatrimestre.

Paquot, T. (2015). L'espace Public. Paris, France: La Dècouverte.

Peñaranda, M. P. (1992). Aproximación a la teoría marxiana desde el pensamiento fenomenológico-existencial de MerleauPonty (I). Logos. Anales del Seminario de Metafísica, 26.

Puche, P. (2019) El gobierno y uso de los bienes comunes según Elinor Ostrom. Cuadernos de Apoyo Mutuo № 2. S.I. España: Promotora cultural malagueña. Ed. Revisada.

Revault d'Allonnes, O. (1977). Creación artística y promesas de libertad. Barcelona. España: Gustavo Gili.

Revault d'Allones, M. (2001) Merleau-Ponty: La Chair du politique (Le bien commun). (French Edition). France: Michalon Editeur. Ed. Kindle.

Sacco, P. L, Ferilli, G. y Tavano, G. (2012) Cultura e sviluppo locale. Verso il distretto culturale evoluto. Bologna, Italia: II Mulino.

Velasco Arroyo, J. C. (2014). Para leer a Habermas. Madrid, España: Alianza Editorial. 